\title{
Universal behaviour of entrainment due to coherent structures in turbulent shear flow
}

\author{
Rama Govindarajan \\ Fluid Dynamics Unit, Jawaharlal Nehru Centre for Advanced Scientific Research, Jakkur, Bangalore 560064, India. E-mail: \\ rama@jncasr.ac.in
}

\begin{abstract}
I suggest a solution to a persistent mystery in the physics of turbulent shear flows: cumulus clouds rise to towering heights, practically without entraining the ambient medium, while apparently similar turbulent jets in general lose their identity within a small distance through entrainment and mixing. From dynamical systems computations on a model chaotic vortical flow, I show that entrainment and mixing due to coherent structures depend sensitively on the relative speeds of different portions of the flow. A small change in these speeds, effected for example by heating, drastically alters the sizes of the KAM tori and the chaotic mixing region. The entrainment rate and, hence, the lifetime of a turbulent shear flow, shows a universal, non-monotone dependence on the heating.
\end{abstract}

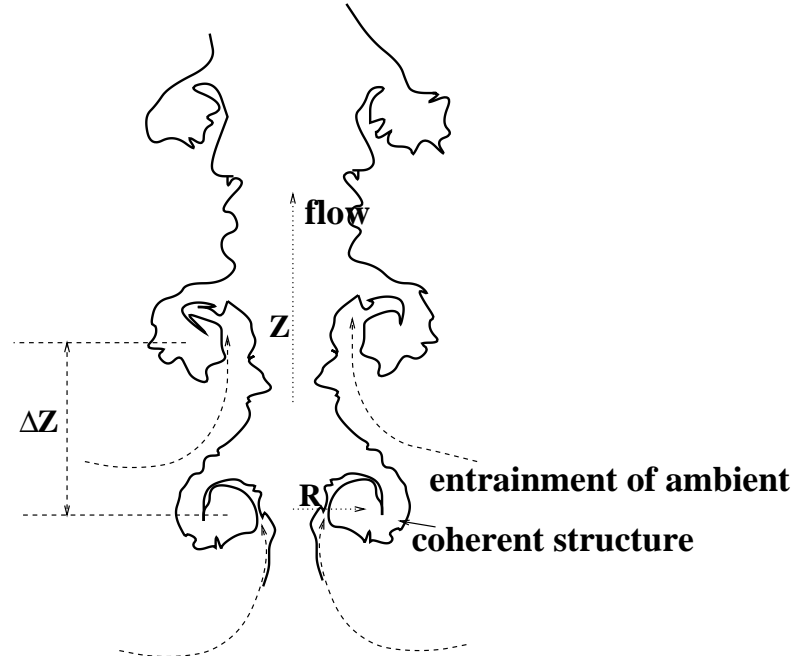

FIG. 1. Schematic sectional view of a turbulent shear flow (a jet). Rotating the figure about the $Z$ axis, the external coherent structures can be seen to be vortex rings. The small scale structures at the edge of the shear flow nibble at the ambient but do not entrain significantly.

Turbulent shear flows [1] in nature and in the laboratory are anisotropic and inhomogeneous on large enough length scales [2,3]. Among their most important properties is entrainment [4], viz., the advection of the ambient non-turbulent medium into the body of the turbulent flow, and the consequent expansion of the turbulent region. Coherent structures [5] at the edges of the turbulent region are crucial to entrainment, but their role in turbulent transport in general is only now beginning to be understood [8]. The entrainment process operates over three length scales [9]. At the large scale, the coherent structures 'engulf' parcels of ambient fluid. The engulfed parcels then 'mingle' with original shear-flow fluid by breaking-up/stretching into smaller scales. Subsequently, the fluids mix at the molecular level when their characteristic sizes are comparable to molecular diffusion scales.

The classic example of a turbulent shear flow is a jet (see Fig. 11), which consists of a turbulent flow injected into a quiescent ambient fluid. Entrainment causes a jet to grow linearly in width, at a rate proportional to its axial velocity (Taylor's hypothesis [10]), as a function of position along its direction of propagation. The width of a plume - a hot jet - grows linearly as well, but faster than a jet [11]. It is therefore deeply puzzling that a cumulus cloud, formed by a rising, hot, moist plume, should entrain practically nothing, and rise to towering heights practically undiluted. Experiments and direct numerical simulations on cloud-like flows 12,13 show, remarkably, that it is the local heating that is responsible for this shut-off of entrainment. This Letter shows that this intriguing phenomenon can be understood by studying the Lagrangian trajectories of fluid particles in a dynamicalsystems treatment of a model jet-like flow, and makes further important predictions.

Our main results are as follows. We establish by quantitative methods on a model that the entrainment and consequently the lifespan of a turbulent shear flow can be dramatically altered merely by tuning the relative velocities (buoyancy) between the coherent structures and the ambient. Surprisingly, a given buoyancy can either increase or decrease entrainment, depending on the spacing between the structures. Secondly, we show that the rate of engulfment is a universal function of the (approriately scaled) relative velocity. Although the inviscid model is incapable of estimating molecular mixing, we are able to make a good assessment of the mingling process using information about the chaos in the system. The results lend themselves to verification by direct numerical simulations and by carefully designed experiments.

I now motivate and construct the model that led to these results. The crucial step in entrainment is the 'engulfing' of ambient fluid by large-scale coherent structures (Fig. 11) which, as experiments as well as direct numerical simulations clearly show, are not destroyed by heating. It is thus vital to understand what heating does do. Such understanding comes not by solving for the flow behaviour in complete detail, but from an economical model with only the essential features. To find such a model, note that the large-scale structures responsible for entrainment in a jet can be approximated (Fig. 1) by 


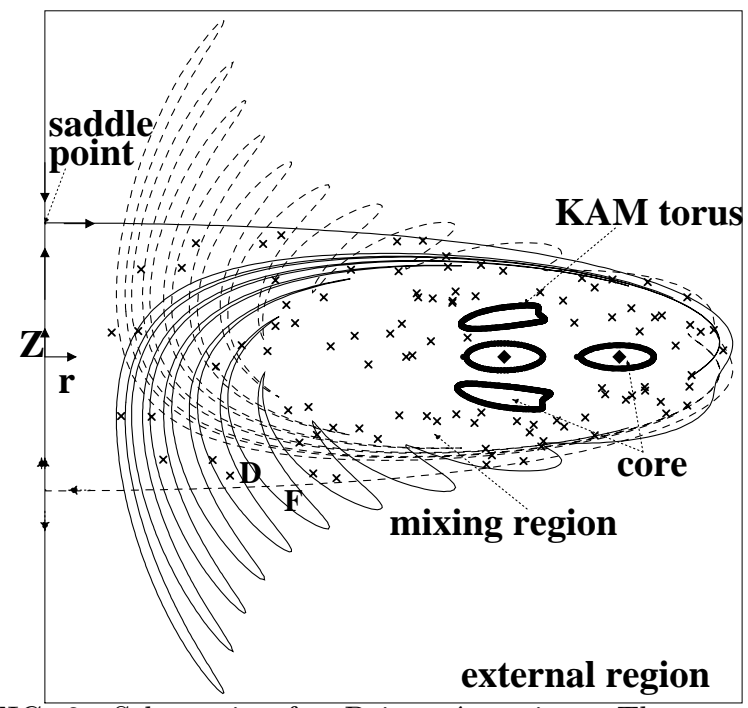

FIG. 2. Schematic of a Poincaré section. The unstable manifold is shown by the solid line, the dotted line shows the stable manifold, and the crosses indicate a sample chaotic trajectory. The phase chosen is that at which the two rings, indicated here by diamonds, are concentric (at $Z=0$ ). The frame of reference moves with the mean axial velocity of the rings.

a stack of vortex rings. Vortical structures in the interior of the jet do not participate in the entrainment process [13. The mixing features of such a flow are largely contained in an even simpler flow, that of a single pair of vortex rings.

We begin with identical coaxial vortex rings of radius $R=1$ and an axial separation $\Delta Z$. Each ring moves forward with the sum of its self-induced velocity $U_{s}$, the induced velocity $U_{i}$ due to the other ring and the prescribed buoyancy velocity $U_{b}$. The rings are taken to be "heated/cooled" if they are buoyant relative to the surrounding fluid, instantaneous acceleration is neglected and the Boussinesq approximation used. The fluid motion then proceeds as follows 114,15 : for each vortex configuration, fluid lagrangian trajectories, obeying Hamiltonian dynamics, may be obtained from the Biot-Savart law. The induced velocities from the vortices lead (a) to leap-frogging of the vortex rings, (b) to portions of the neighboring flow being trapped forever within KAM tori, and (c) to complex, often chaotic trajectories elsewhere, both within the jet and extending into the ambient. The dynamics in the last region is responsible for entrainment and subsequent mingling of ambient fluid with original jet fluid [16,17]. These features may be seen on the Poincaré map (Fig. 2) in $r-Z$ space, where $r$ and $Z$ are the radial and axial coordinates respectively. The engulfment may be obtained quantitatively 16] from the tangling of manifolds in this map. The flow being axisymmetric, regions that appear as lobes in Fig. 2 are funnel-shaped structures of equal volume. During each half-cycle of leapfrog, the volume of fluid contained in one funnel (denoted by $F$ in the figure) is entrained into the turbulent flow from

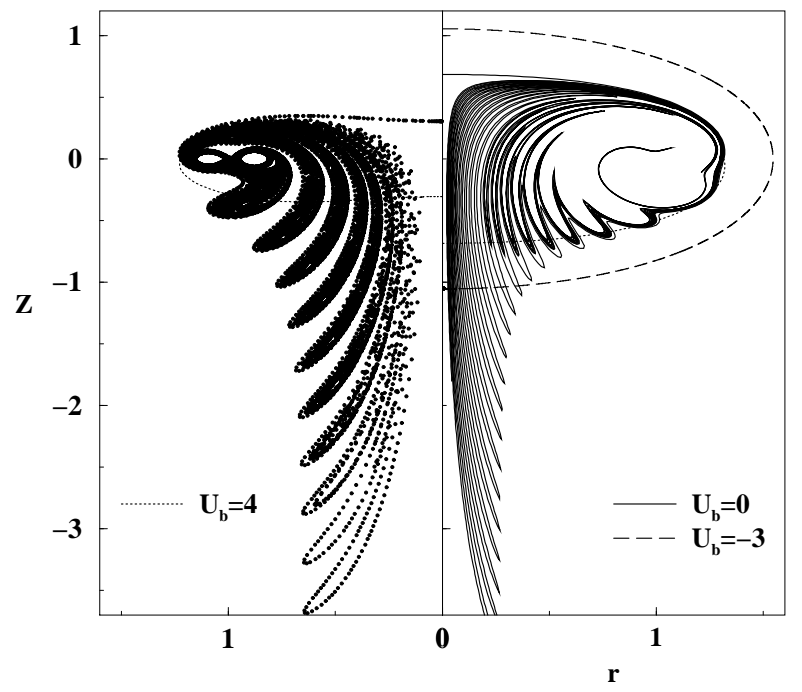

FIG. 3. The unstable manifold for small ring spacing $(\Delta Z=0.22)$. The stable manifold for $U_{b}=4$ as $t \rightarrow \infty$ is shown by the dotted line. (The self-induced ring velocity $U_{s} \sim 4.5$, so $\left.U_{b} / U_{s} \sim 1\right)$.

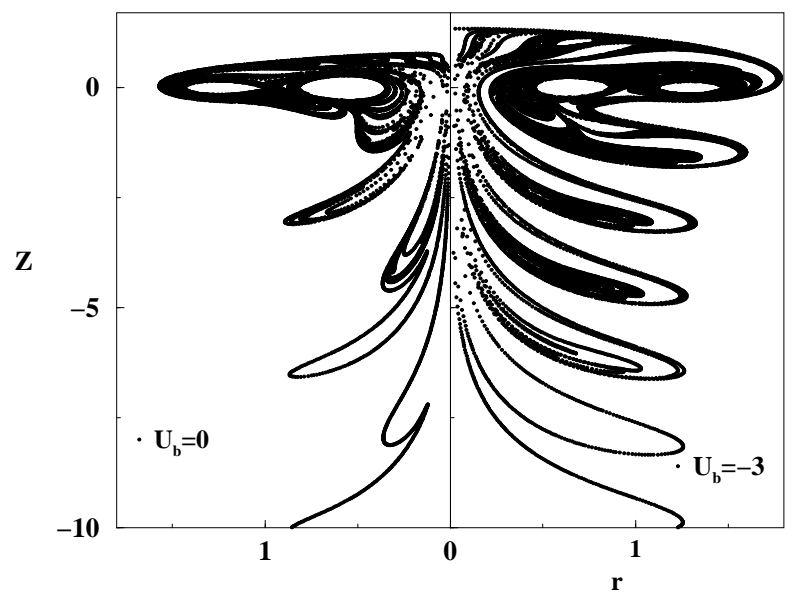

FIG. 4. The unstable manifold for a large ring spacing $(\Delta Z=1.00)$.

the ambient, while an equivalent volume $(D)$ is detrained from within the mixing region and dumped into the ambient [16]. The quantity engulfed in a fraction $f$ of a cycle is equal to $2 f F$. The toroidal volume and the circulation of the rings are held respectively at $0.2 \pi^{2}$ and $4 \pi$, fixing the basic velocity and time scales.

In the absence of buoyancy, the present predictions agree with the results of [18], corresponding to a small $\Delta Z$, for a leap-frogging vortex pair. In addition, we find a maximum in the engulfment rate (proportional to the Melnikov function [19]) as a function of the maximum separation $\Delta Z$ (inversely related to the frequency of leapfrog), which agrees with [20]. Our main results emerge when 'buoyancy' is switched on: for small ring spacing $(\Delta Z=0.22)$, the entrainment is greatly enhanced (Fig. 3) when the direction of buoyancy is such as to speed up the rings. In contrast, when the rings are slower, the 


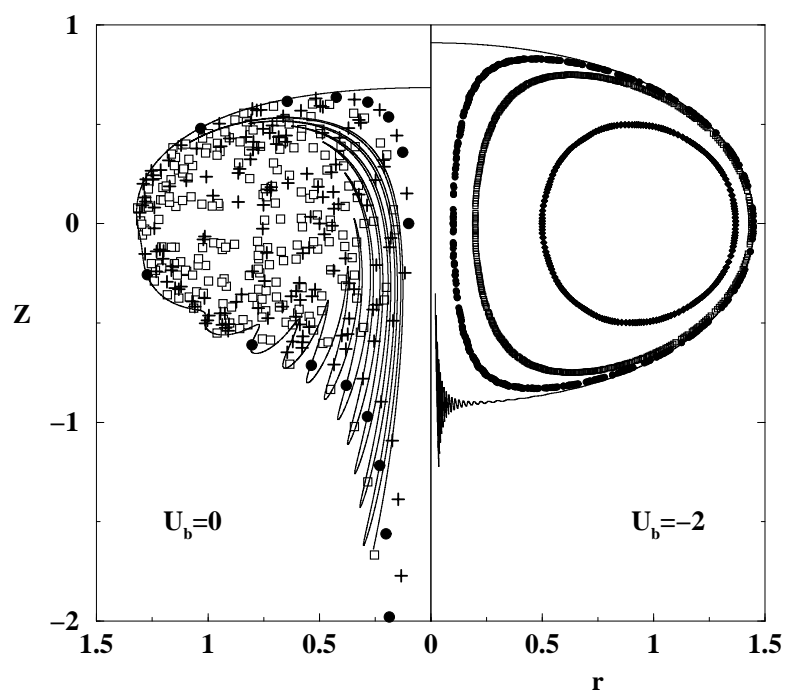

FIG. 5. Trajectories of fluid particles when $U_{b}=-2$. The three symbols represent three different starting locations.

entrainment is killed off almost completely. Surprisingly, as $\Delta Z$ increases, the role of $U_{b}$ is progressively reversed, until, at $\Delta Z=1.0$ (Fig. (1), the engulfment is inversely related to $U_{b}$. The extent of chaos within the region of influence of the vortical structures provides an estimate of the 'mingling' between original and ambient fluid. It is seen in Fig. 5 that for $\Delta Z=0.22$, when $U_{b}=-2$, a major portion of the region of interest consists of a series of imbedded KAM tori, which make any degree of mingling unlikely. At $U_{b}=-1$ (not shown) slow mixing takes place, while the chaotic nature of the flow at $U_{b}=0$ indicates that any entering fluid will follow a space-filling trajectory, undergo stretching into smaller scales and mingle with the original fluid, resulting in a dilution of the latter. Fig. 6 shows the dependence of $F$ on the buoyancy for different values of ring spacing. For the highest spacing, due to the extreme tangling of the manifolds, the computations were reliable only for the range of $U_{b}$ shown. When $\Delta Z$ is about half the radius, the engulfment decreases significantly whether the fluid is heated or cooled. For each ring spacing the engulfment displays a maximum at some value of the buoyancy velocity $U_{b}$. Table 1 shows characteristic axial velocities for different $\Delta Z$. Here $\hat{U}_{i}$ is the maximum axial velocity, scaled by $\bar{U}_{s}$, induced by one of the rings on the other during a given cycle, $\left(\hat{U}_{i}\right.$ would also be the typical velocity induced by one of the rings on a non-vortical fluid element at the same distance.) $\left[\hat{U}_{b}\right]_{\text {opt }}$ is the buoyancy velocity required to maximize engulfment at a given $\Delta Z$. The last column in the table is independent of $\Delta Z$, which suggests that we define a velocity ratio $U_{r}$ :

$$
U_{r} \equiv \frac{U_{i}}{U_{i}+U_{b}+c \bar{U}_{s}}
$$

whose numerator is a measure of the velocity of the induced motion in the surrounding fluid, and denominator

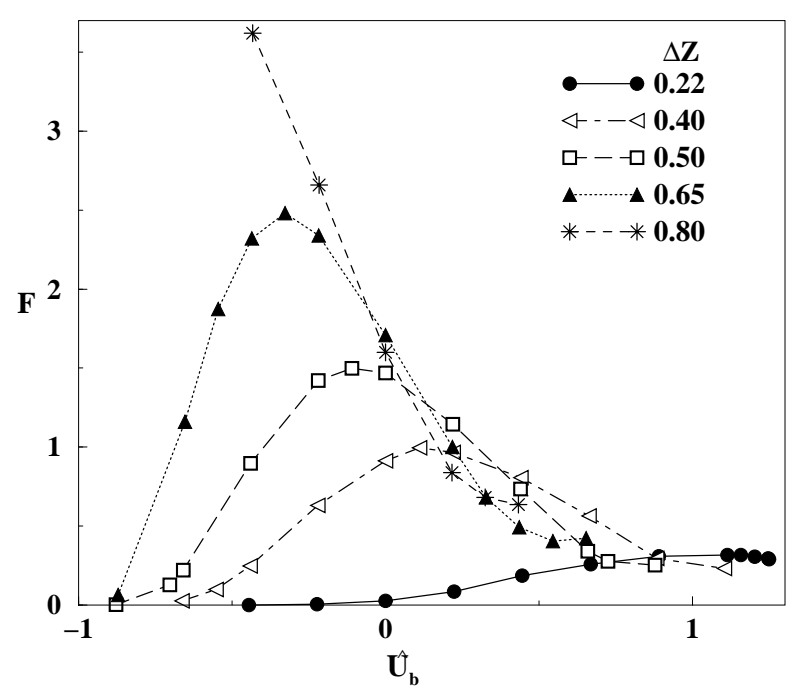

FIG. 6. Entrainment volume as a function of buoyancy velocity imparted to the rings. $\hat{U}_{b}=U_{b} / \bar{U}_{s}$ is the buoyancy velocity scaled by the average self-induced velocity of a ring.

represents the total ring speed. The constant $c(1.55$ here) depends on the ratio of the ring thickness to the toroidal radius, as well as on the particular measures of the self and mutually induced velocities used. Since the period $\tau$ of a leapfrog is vastly different for different ring spacings, the rate of engulfment $E \equiv F / \tau$, would be a more appropriate measure for comparison. This quantity is plotted in Fig. 7 as a function of the velocity ratio $U_{r}$.

Table 1

\begin{tabular}{|c|c|c|c|c|}
\hline$\Delta Z$ & $\hat{U}_{i}$ & {$\left[\hat{U}_{b}\right]_{\mathrm{opt}}$} & $\bar{U}_{s}$ & $\hat{U}_{i}-\left[\hat{U}_{b}\right]_{\mathrm{opt}}$ \\
& & & & \\
0.22 & 2.676 & 1.130 & 4.493 & 1.55 \\
0.40 & 1.689 & 0.128 & 4.531 & 1.56 \\
0.50 & 1.450 & -0.110 & 4.556 & 1.56 \\
0.65 & 1.236 & -0.327 & 4.591 & 1.56 \\
0.80 & 1.109 & -0.433 & 4.620 & 1.54 \\
\hline
\end{tabular}

The rate of entrainment is seen to be a universal function of the velocity ratio, i.e., the parameter which determines the entrainment rate is the ratio of the motion induced in the surroundings to the speed of the coherent structures. It is observed that the entrainment when the rings are much faster than the surroundings (extreme left of Fig. (7) levels off at a small value. This feature will be investigated in detail elsewhere.

In the simulations of [13], the structure spacing is reduced upon heating from about $\Delta Z=1$ to $\Delta Z=0.3$. The ratio $U_{r}$ in the present scales is about 0.45 in the pre-heating zone, where we expect a significant level of entrainment; and about 0.7 in the heating zone, where entrainment should drop almost to zero; which concur with what the simulations show. In a lab jet (or in a cloud), the entire turbulent flow would be heated with 


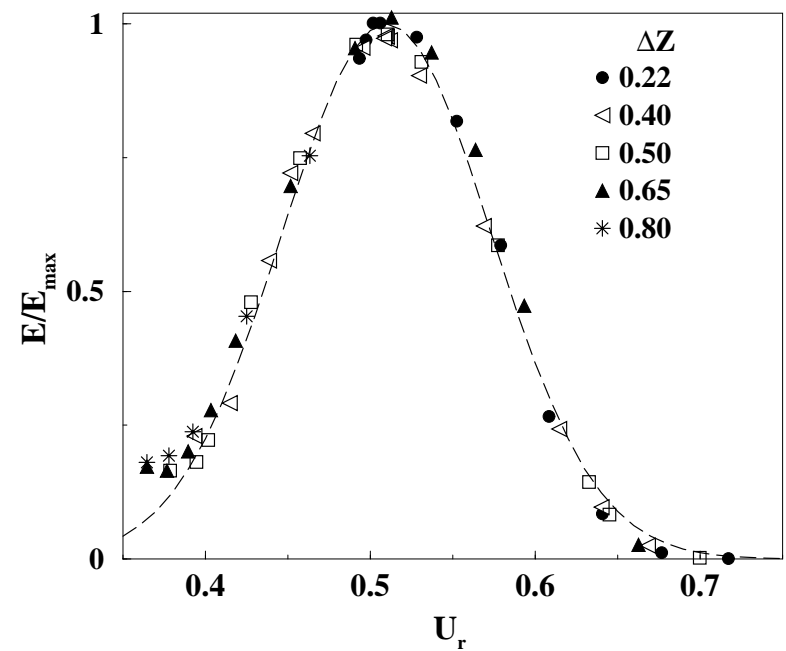

FIG. 7. Dependence of the entrainment rate on the velocity ratio. The fit $E / E_{\max }=\exp \left\{-\left[\left(U_{r}-U_{\text {ropt }}\right) / \sigma\right]^{2}\right\}$ is shown by the dashed line. The fitting parameters have the numerical values $E_{\max }=4.3$ and $\sigma^{2}=0.09$ here, which will change with the circulation prescribed.

respect to the ambient, not the structures alone. Moreover, the rings would be of unequal sizes and strengths. We have examined these situations: when the entire internal fluid, or a selected portion of it, is given the same buoyancy as the rings, the results are very close to those shown above, demonstrating that the important parameter is the relative velocity between the structures and the external flow, and not the state of buoyancy of the interior fluid. Inequalities in the rings result in one ring becoming dominant over the other, but have very little qualitative effect on the results.

In summary, it is known from observations that the entrainment in heated plumes is higher than in unheated jets, while that in (heated) clouds is much lower. This is the first computation to our knowledge that addresses this apparent contradiction, obtains entrainment quantitatively albeit for a model heated flow, and shows that an imposed relative buoyancy can either increase or decrease the entrainment. For example in Fig. 7, when $\Delta Z=0.5$, the maximum entrainment occurs when $U_{b}=0$, speeding-up or slowing-down the rings would both decrease entrainment. For small values of structure spacing, the entrainment is directly related to the buoyancy (with the sign as defined here), while while at large spacing they are inversely related. The data collapse seen in Fig. 7 could not have been anticipated from intuition, and much less from an examination of the tangling of the manifolds. The funnel shapes and the convolutions they undergo are often qualitatively different in each case (they sometimes even have two or three sub-lobes per half-cycle), and so is the volume they entrain, but the rate of entrainment obeys a very simple relationship. Implicit in the data collapse is the remarkable observation that the maximum rate of engulfment (for a given circulation and geometry) is independent of the system frequency. An increase in the engulfment of external fluid due to buoyancy is shown to be accompanied by an enlargement of the chaotic region, resulting in increased mingling. In closing, note that we have neglected viscous effects as well as the complicated manner in which heating/cooling is bound to affect a turbulent shear flow, by altering the geometry, density and orientation of the coherent structures, which would in turn affect entrainment. Including these would be natural directions for future research. In order to test the present predictions and to strengthen our understanding the effect of heat, systematic experiments and direct numerical simulations are called for. The ideal experiment would be on a laboratory-generated isolated pair of vortex rings, whose circulations could be estimated and which could be heated or cooled over some axial distance.

I thank A. Leonard, S. Wiggins, R. Narasimha and K. R. Sreenivas for discussions and useful suggestions. This work is funded by the AR\&DB, India.

[1] S. Pope Turbulent Flows, Cambridge Univ. Press, (2000).

[2] F. Toschi et al., Phys. Rev. Lett. 85, 1436 (2000).

[3] S. Kurien et al., Phys. Rev. E 61, 407 (2000).

[4] A. Roshko, AIAA J. 14, 1349, 1976.

[5] F. Spineanu \& M. Vlad, Phys. Rev. Lett. 84, 4854 (2000).

[6] P. Jung et al., Phys. Rev. E 61, 2095 (2000).

[7] F. Waleffe, Phys. Rev. Lett. 81, 4140 (1998).

[8] Z. Toroczkai et al., Phys. Rev. Lett. 80, 500 (1998).

[9] R. Narasimha \& L. Venkatakrishnan, Proc. Eighth Asian Cong. Fluid Mech. 275 (1999).

[10] B.R. Morton, G.I. Taylor \& J.S. Turner, Proc. Roy. Soc. Lond. A 234, 1 (1956).

[11] K.R. Sreenivas \& A.K. Prasad, Phys. Fluids, 12, No.8, 2101 (2000).

[12] G.S. Bhat \& R. Narasimha, J. Fluid Mech. 325, 303 (1996).

[13] A.J. Basu \& R. Narasimha, J. Fluid Mech. 385, 199 (1999).

[14] K. Shariff \& A. Leonard, Ann. Rev. Fluid Mech. 24, 235 (1992).

[15] R. Govindarajan, A. Leonard \& S. Wiggins in Lecture Notes in Physics, ed. C.-H. Bruneau, Springer, 482 (1998).

[16] V. Rom-Kedar, A. Leonard \& S. Wiggins, J. Fluid Mech. 214, 347 (1990).

[17] For a detailed discussion on the use of dynamical systems in this context, see T. Dombre et al., J. Fluid Mech. 167, 353 (1986) and 16].

[18] A. Pentek, T. Tel \& Z. Toroczkai J. Phys. A 28, 2191 (1995).

[19] S. Wiggins, Introduction to Applied Nonlinear Dynamical Systems and Chaos, Vol. 2, TAM, Springer-Verlag (1990).

[20] V. Rom-Kedar \& A.C. Poje, Phys. Fluids 11, No. 8, 2044 (1999). 